Polymer Journal, Vol. 39, No. 5, pp. 435-441 (2007)

(C) 2007 The Society of Polymer Science, Japan

\title{
Effect of Solvent on Morphological and Structural Change of Cellulose under Ball-Milling
}

\author{
Mariko AGo, ${ }^{1}$ Takashi Endo, ${ }^{2, \dagger}$ and Kunihiko OKAJIMA ${ }^{1}$ \\ ${ }^{1}$ The Faculty of Engineering, Tokushima Bunri University, 1314-1 Shido, Sanuki City 769-2193, Japan \\ ${ }^{2}$ National Institute of Advanced Industrial Science and Technology, 2-2-2, Hirosuehiro, Kure 737-0197, Japan
}

(Received August 22, 2006; Accepted February 6, 2007; Published March 23, 2007)

\begin{abstract}
Cellulose derived from cotton was ball-milled with some hydrophilic and hydrophobic solvents such as water, toluene, 1-butanol and also without solvent (dry state), and effect of the solvents on morphological and structural changes of the cellulose were investigated. The original fibrous cellulose was changed to the aggregated globular particles by water and dry state ball-milling, plate-like particles by toluene processing, and a mixture of rugged globular particles and plate-like particles by 1-butanol processing. The globular particles prepared without solvent showed fairly low crystallinity and their inter- and intra-molecular hydrogen bonds were deformed, leading to an increase in free hydroxyl groups. The globular particles prepared with water exhibited a mixture of crystalline structure as cellulose I, cellulose II and non-crystalline state. In the case of the products with toluene, the plate-like particles significantly retained their original crystalline structure and hydrogen bonds. The 1-butanol processing seems to give the particles consisting of cellulose I and small amounts of either non-crystalline or cellulose II. The cellulose/dye stuff mixtures under the presence of the above solvents were prepared using 4-aminoazobenzene to investigate the differences in the surface property of the particles prepared by the ball-milling processing. Characteristic color development of each cellulose particles was clearly distinguished depending on the effect of the solvent (cinnabar; without solvent, yellow; toluene and yellowish-green; water or 1-butanol). [doi:10.1295/polymj.PJ2006096]

KEY WORDS Cellulose / Ball-Milling / Hydrogen Bonds / Crystallinity / Hydrophobic Interaction /
\end{abstract}

It has been shown that a typical hydrophilic solvent, water causes change in the long-periodicity of regenerated cellulose fiber at $30-40 \%$ water content ${ }^{1}$ and the amorphous cellulose prepared by a ball-milling treatment reorganizes effectively to cellulose II crystalline by ball-milling with a specific amount of water; $30 \mathrm{wt} \% .^{2}$ Such apparent concentration-specific structural change in cellulose is also observed in measurements of ${ }^{1} \mathrm{H}$ spin-lattice relaxation of cellulose for a cellulose-water system as a function of concentration of water. ${ }^{3}$ Molecular mobility of cellulose increases and reaches a maximum around $30 \mathrm{wt} \%$ of water content, at which the water works as a thermo-plasticity agent on the cellulose in the system. ${ }^{2,3}$

On the one hand, it has been also shown that hydrophobic solvents affect the morphology and structure of cellulose: G. N. Marchenko et al. ${ }^{4}$ reported on wood pulp and cotton that dichloromethane (DCM) facilitated an intra-fibril swelling and gave some changes in a absorption of $\mathrm{CH}_{2}$-vibration (IR; $1380 \mathrm{~cm}^{-1}$ ) with no practical change in X-ray crystallinity. A concentration-specific interaction of toluene with a regenerated cellulose membrane with high planar orientation has been proved to show that the orientation of (110) plane in cellulose crystal preferentially lowers around $20-40 \mathrm{wt} \%$ of toluene content, elucidated by X-ray diffraction patterns. ${ }^{5}$ These observations show that cellulose molecules can transform spontaneously into other aggregate states under the existence of the solvent with apparent specific content.

Recently, Yamane et al. revealed that structural anisotropy of cellulose crystals derived from molecular anisotropy give rise to hydrophilic and hydrophobic crystal planes by means of contact angle of water droplet and computer simulations. ${ }^{6}$ These results clearly suggest that both hydrophobic and hydrophilic parts must exist in cellulose and each part is possible to interact with hydrophobic and hydrophilic solvents, respectively.

Therefore, it is quite interesting to investigate how and where a foreign substance is accommodated with aids of solvents present in cellulose under outer force such a ball-milling which will enables us to prepare a new class of cellulose composites. In this connect, we used a dye-stuff as a foreign substance since dyes have been utilized to detect the void architecture of surface, ${ }^{7}$ crystal surface, ${ }^{8}$ and polymer chain orientation $^{8,9}$ of cellulose.

Thus, in this report morphological and structural changes of cellulose affected by the type of solvent under ball-milling were examined and the surface (or interfacial) property of cellulose was discussed

${ }^{\dagger}$ To whom correspondence should be addressed (Tel: +81-823-72-1111, Fax: +81-823-72-1990, E-mail: t-endo@aist.go.jp). 
based on the difference in color development observed by a dye stuff adsorbed.

\section{EXPERIMENTAL}

\section{Cellulose Sample}

Original fibrous cellulose (CF11, Whatman Co., Ltd.), derived from cotton linters, was used in this study as microcrystalline cellulose (Cellulose $\mathrm{I}_{\beta}$ ), with a crystallinity for Cellulose I of $91 \%$ determined by wide angle X-ray diffraction (WAXD). ${ }^{10}$ The degree of polymerization, $\mathrm{DP}_{\mathrm{v}}=220,{ }^{2}$ corresponding to the level off polymerization degree, was determined by the intrinsic viscosities of a copper-ethylenediamine solution. The cellulose sample was vacuum-dried at $60{ }^{\circ} \mathrm{C}$ for $8 \mathrm{~h}$ before use.

\section{Ball-Milling Treatment}

Cellulose with $40 \%(\mathrm{v} / \mathrm{w})$ of water, toluene or 1-butanol and without solvent was submitted to a ball-milling for $4 \mathrm{~h}$ or $8 \mathrm{~h}$ at $200 \mathrm{rpm}$ using an apparatus of Pulverisette-5 (FRITSCH Co., Germany). The processing was carried out with repeated milling and pausing of $10 \mathrm{~min}$ each to avoid overheating of the cellulose and solvent in the milling pots.

\section{Cellulose and Dye Stuff Mixture}

To the mixtures obtained after milling for $4 \mathrm{~h}$ with each solvent was added $0.1 \mathrm{wt} \%$ of 4 -aminoazobenzene (AAB) as a dye stuff (Wako Chem. Co. Ltd., Japan) which dissolves in the three kinds of solvents used in this experiment and the mixture of the cellulose with solvent and $\mathrm{AAB}$ was ball-milled again for $4 \mathrm{~h}$. The cellulose/AAB mixtures were washed well with toluene to remove excess of $\mathrm{AAB}$, and subjected to optical microscopic observation. After the processing, all samples were dried at $60^{\circ} \mathrm{C}$ in a vacuum oven for $48 \mathrm{~h}$ and transferred to a vacuum desiccator to avoid absorption of moisture from atmosphere.

The samples were characterized by WAXD and FT-IR spectroscopy and scanning electron microscopy (SEM). X-Ray diffraction patterns of samples were measured by reflection method and recorded on a X-ray diffractometer with scintillation counter (RINT 2000 diffractometer, RIGAKU, Japan; $\mathrm{Cu}-\mathrm{K} \alpha$ at $40 \mathrm{kV}$ and $24 \mathrm{~mA}$ ) to evaluate the crystalline polymorph and the crystalline index of cellulose. ${ }^{10} \mathrm{IR}$ spectra were obtained using a JASCO FT-IR-670 plus (Japan) with analyzing software. To prepare $\mathrm{KBr}$ pellets, about $2 \mathrm{mg}$ of the samples were taken, grinded for 5 min together with about $100 \mathrm{mg}$ of $\mathrm{KBr}$ (FT-IR grade, JASCO, dried). The pellets were pressed to produce transparent disks with $2 \mathrm{~mm}$ in diameter. The samples for IR measurement were significantly dried at $80^{\circ} \mathrm{C}$ in vacuo before the measurement. The

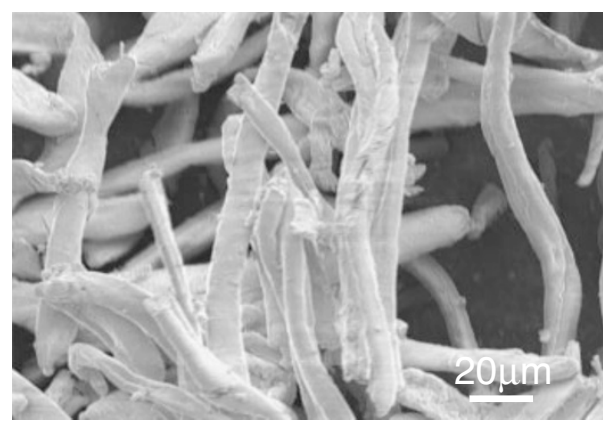

Figure 1. SEM micrograph of the original cellulose.

particle size and their morphologies of the samples were directly estimated by SEM (KEYENCE VE7800, Japan) observation.

\section{RESULTS AND DISCUSSION}

\section{Ball-Milling of Cellulose with Solvents}

Figure 1 shows the SEM micrograph of the original cellulose having fibrous morphology with 100-200 $\mu \mathrm{m}$ in length and $10-20 \mu \mathrm{m}$ in diameter. The SEM micrographs of the surface of milled celluloses at an early stage of the ball-milling processing and the final product at small window without solvent (a) and in presence of water (b), toluene (c) and 1-butanol (d) are shown in Figure 2.

The original fibrous cellulose was milled enough to convert to the aggregated globular particles with a diameter of 5-10 $\mu \mathrm{m}$ without solvent under a dry state even at an early stage of the processing (Figure 2a), resulting in a fairly low crystallinity, as shown by $\mathrm{X}$-ray diffraction profile (Figure 3a). The ball-milling with water caused much cracks in the concentric circle direction with tilt angle against the fiber axis on the original cellulose fibers (Figure 2b). The aggregated globular particles were finally obtained after the processing (Figure 2b, small window). The profile of $\mathrm{X}$-ray diffraction of the product revealed that the crystalline structure was partly converted to cellulose II showing its reflections around $2 \theta=12$ and $20^{\circ}$ and retained some cellulose I crystal with reflections around $2 \theta=15,17$ and $23^{\circ}$, with showing a halo pat$\operatorname{tern}^{11}$ (Figure $3 \mathrm{~b}$ ). In the case of toluene processing, no deep cracks on the fiber were observed at an early stage of the processing (Figure 2c). The plate-like particles were peeled off from the surfaces of the fibrous cellulose, having a diameter of $5 \mu \mathrm{m}$ and thickness of less than $1 \mu \mathrm{m}^{15}$ and the fracture surface of them was brittle (Figure 2c). That is, it is destructed in the concentric circle direction, in contrast to the case of the water processing. The plate-like particles considerably maintained crystallinity as cellulose I, even after the prolonged processing and did not form cellulose II 

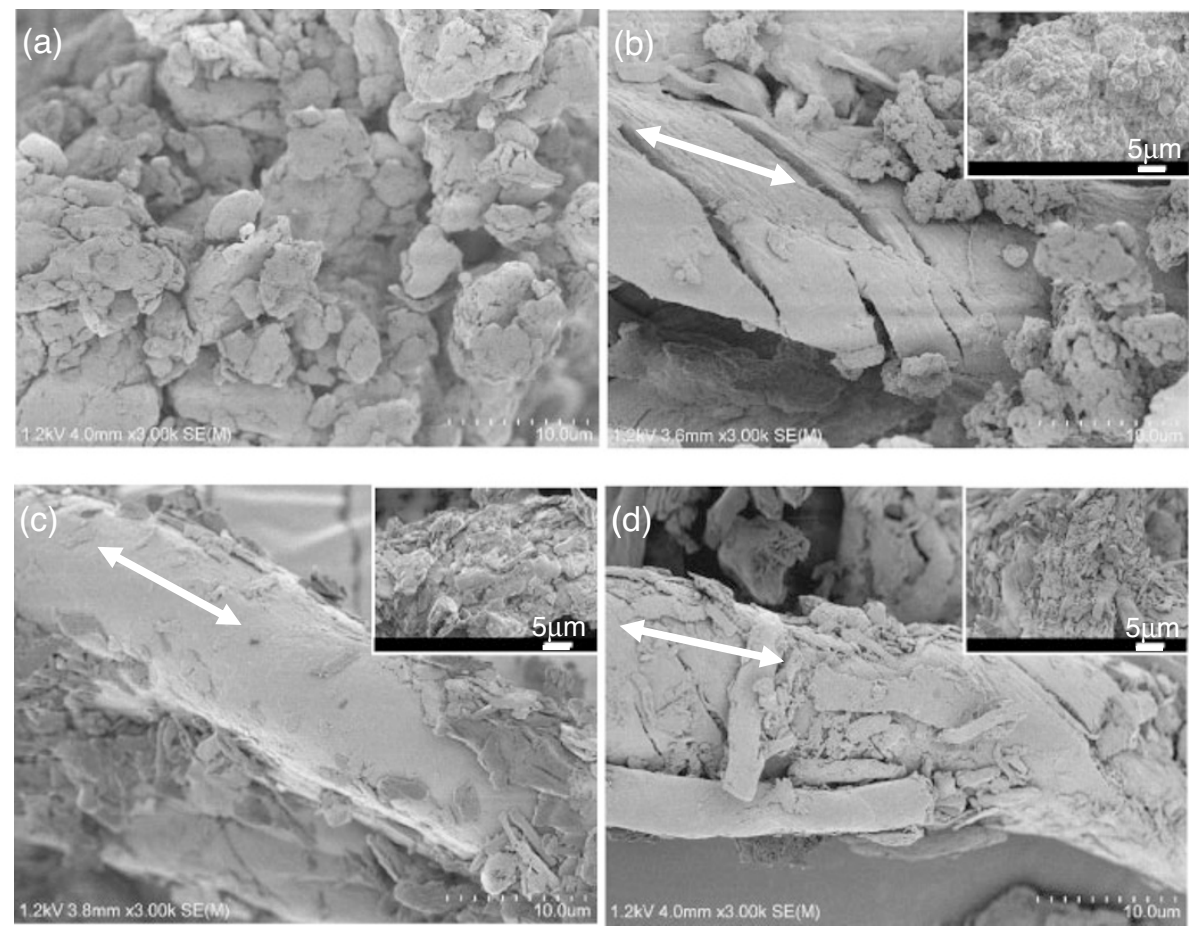

Figure 2. SEM micrographs of the milled cellulose at an early stage of ball-milling processing and at final stage in small window, without solvent (a), and in presence of water (b), toluene (c), and 1-BuOH (d). The arrow in the pictures shows the fiber direction.

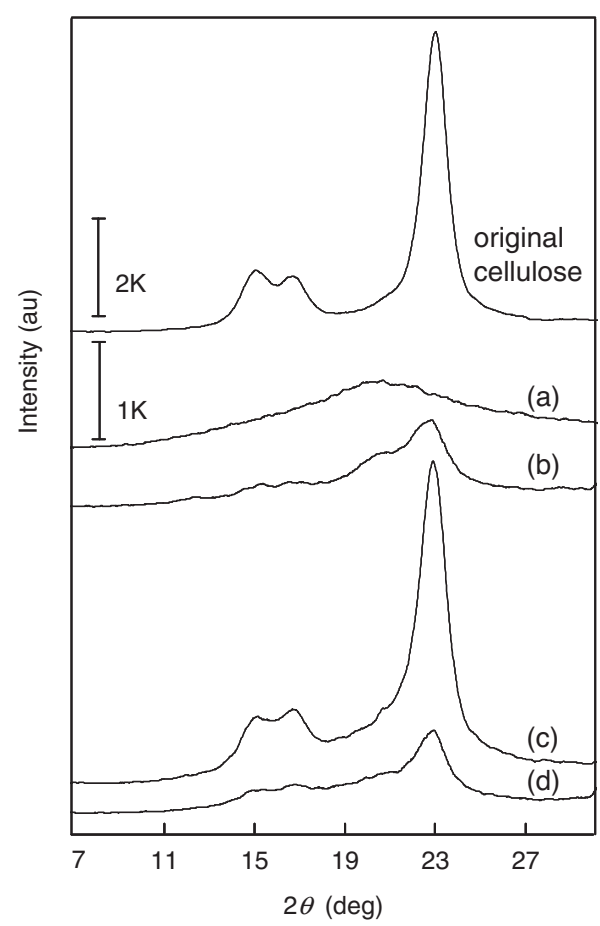

Figure 3. WAXD profiles of the original cellulose and the milled cellulose, without solvent (a) and in presence of water (b), toluene (c), and 1-BuOH (d).

(Figure 3c). The particle morphology under the processing with 1-butanol seem to be a mixture of globular and plate-like particles and its original crystalline structure was fairly kept, but with some amorphous (Figure 2d and Figure 3d). Note that no $\mathrm{DP}_{\mathrm{v}}$ change during the ball-milling was observed in the case of cellulose/solvent systems.

Such morphological and crystalline structural changes seem to be attributed by the difference of interaction between the solvent and cellulose. It is possible that a lot of hydrogen bonds formed in the crystalline structure of cellulose were changed under the ball-milling processing. In order to understand the relationship between morphology of the fibrous cellulose and structure of cellulose, hydrogen bonds in the particle was analyzed by means of IR method (Figure 4-6). The difference spectra between the products and the original in the regions of $\mathrm{O}-\mathrm{H}$ stretching from 3000 to $4000 \mathrm{~cm}^{-1}$ and $\mathrm{C}-\mathrm{O}$ stretching vibrations from 800 to $1400 \mathrm{~cm}^{-1}$ are compared among all products in Figures 5 and 6, respectively.

The difference spectra in each region of $\mathrm{O}-\mathrm{H}$ stretching and $\mathrm{C}-\mathrm{O}$ stretching were similar between the products obtained without solvent and by the water processing. The $\mathrm{O}-\mathrm{H}$ stretching bands of the ballmilled ones clearly shifted toward higher wave numbers around $3500-3600 \mathrm{~cm}^{-1}$, indicating an increase in free hydroxyl groups or the ones with weak hydrogen bonds, ${ }^{18}$ while the bands at 3267/3270 and 3335/ $3347 \mathrm{~cm}^{-1}$ were decreased (Figure 5a and $5 \mathrm{~b}$ ). According to the literatures, ${ }^{18-21}$ they are assigned to inter-molecular hydrogen bond at $\mathrm{O}(6) \mathrm{H}-\mathrm{O}(2)$ and intra-molecular hydrogen bond at $\mathrm{O}(3) \mathrm{H}-\mathrm{O}(5)$. In $\mathrm{C}-\mathrm{O}$ stretching region, the bands at 1034/1030 and $1058 / 1060 \mathrm{~cm}^{-1}$ were decreased by the processing 
of both samples. These peaks are assigned to $\mathrm{C}(6)-$ $\mathrm{O}(6)$ and $\mathrm{C}(3)-\mathrm{O}(3)$ stretching, respectively. ${ }^{22}$ These results suggest that inter- and intra-molecular hydrogen bonds of the products were broken by the ballmilling.

The difference spectra of the products obtained by the toluene or 1-butanol processing were similar to

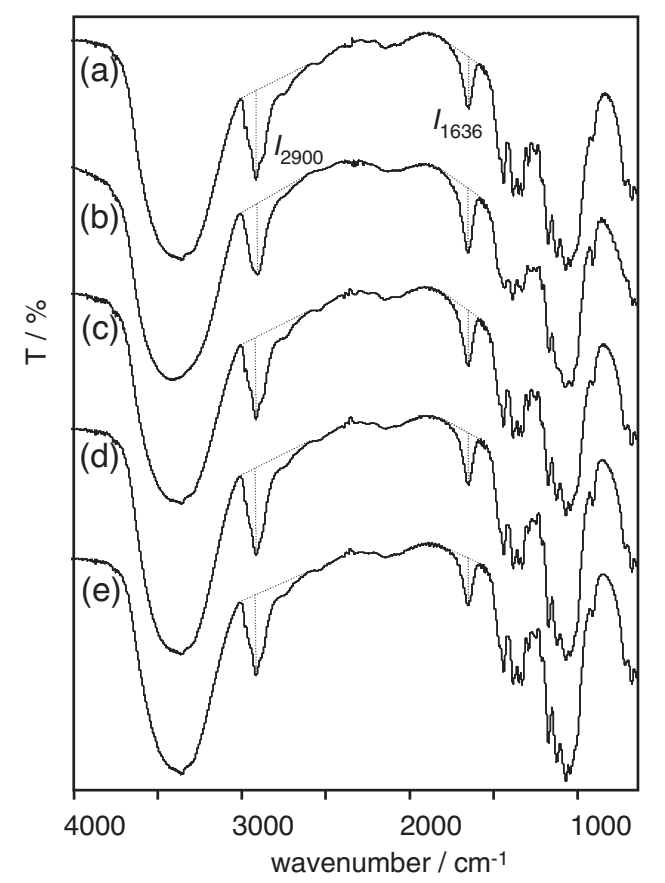

Figure 4. FT-IR spectra of the original cellulose (a) and the milled cellulose, without solvent (b) and in presence of water (c), toluene (d), and 1-BuOH (e).
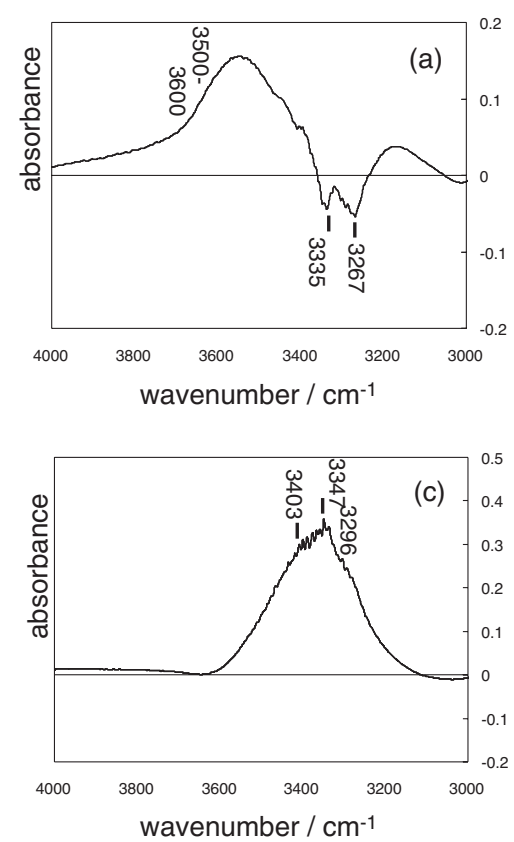

each other in both regions of $\mathrm{O}-\mathrm{H}$ and $\mathrm{C}-\mathrm{O}$ stretching (Figure $5 \mathrm{c}$ and $5 \mathrm{~d}$, Figure $6 \mathrm{c}$ and $6 \mathrm{~d}$ ). The absorption bands at $3500-3600 \mathrm{~cm}^{-1}$ decreased, while the bands at $3296 / 3282$ and $3347 / 3348 \mathrm{~cm}^{-1}$ still existed in contrast to the products obtained without solvent or by the water processing. In $\mathrm{C}-\mathrm{O}$ stretching region, the bands at $1038 / 1035$ and $1059 \mathrm{~cm}^{-1}$ were considerably more intense than that of the original. All the results indicate that the products by toluene and 1butanol processing kept fairly the original conformations stabilized by hydrogen bonds. ${ }^{23}$ It is found that the characteristic hydrogen bonds retain in the particles depending on their morphology.

It should be noted that a small amount of carboxyl groups were contained in the original cellulose, giving a corresponding peak at $1636 \mathrm{~cm}^{-1}$. We affirmed that the peak shifted to far lower wave number by $\mathrm{NaOH}$ treatment. The relative intensity ratio of the peak of carboxyl group $\left(I_{1636} / I_{2900}\right)$ increased to 1.5 and 1.1 times than that of original cellulose by the ballmilling without solvent and with water, respectively (Figure 4). On the other hand, the increase of the ratio was not observed in the products by the toluene and 1-butanol processing. It almost means that the degradation of cellulose chains occurred under ball-milling without solvent. Note that $\mathrm{OH}$ deformation peak for the absorbed water was observed near the above carboxyl bands $\left(c a .1646 \mathrm{~cm}^{-1}\right)$ and the band strength lowered by vacuum drying.

To understand the solvent effect on the morphological and the structural changes under the ball-milling, the characteristic feature of cellulose structure will be
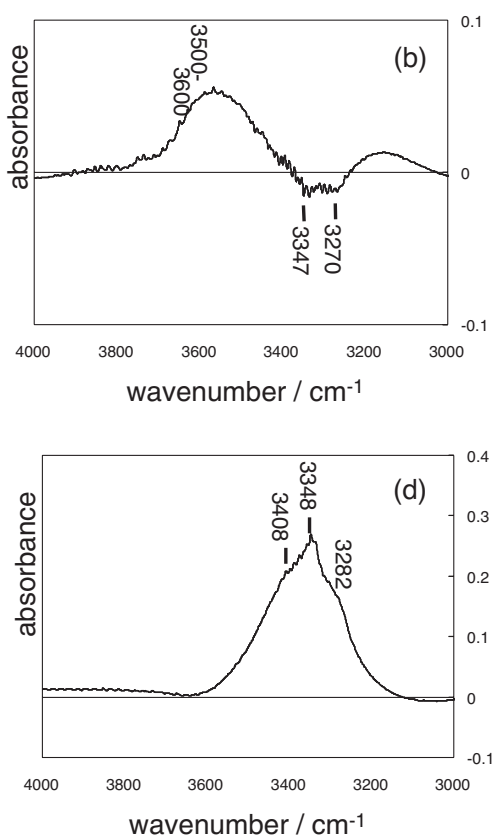

Figure 5. The difference FT-IR spectra between the ball-milled products and the original, in the hydroxyl region $\left(4000-3000 \mathrm{~cm}^{-1}\right)$, without solvent (a) and in presence of water (b); toluene (c) and 1-BuOH (d). (The peak at $2900 \mathrm{~cm}^{-1}$ is offset). 

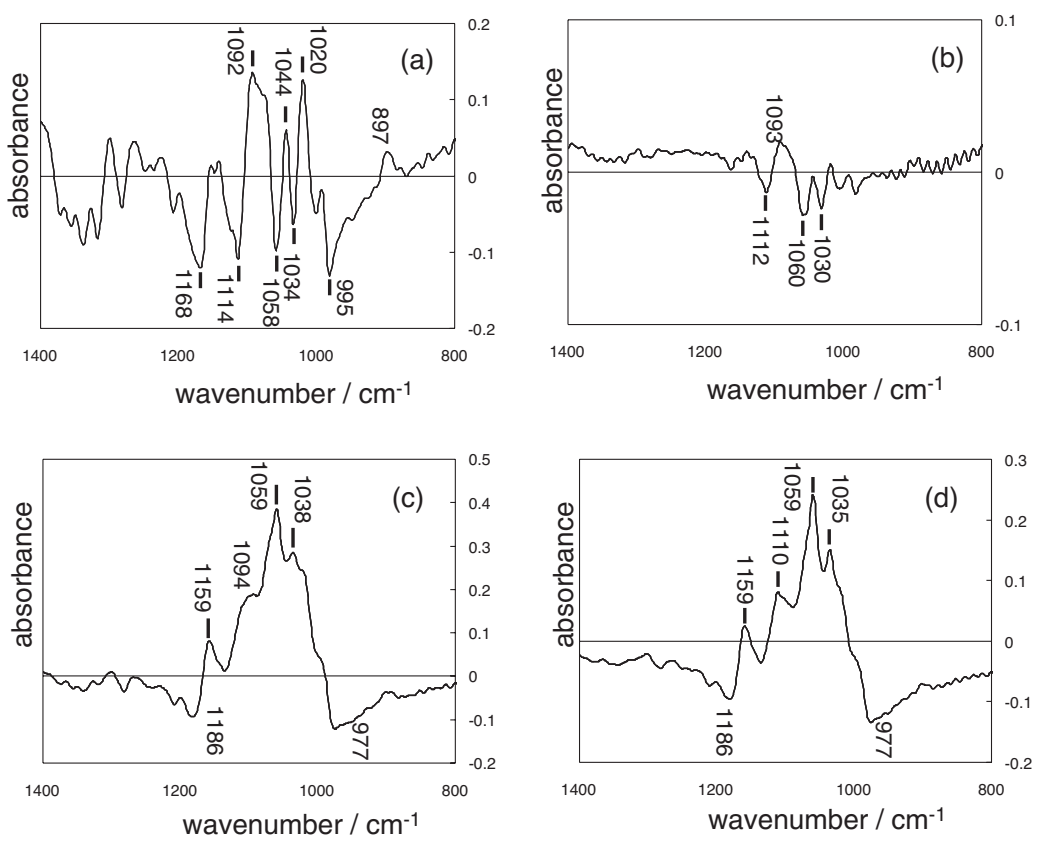

Figure 6. The difference FT-IR spectra between the ball-milled products and the original in the C-O region $\left(1400-800 \mathrm{~cm}^{-1}\right)$, without solvent (a) and in presence of water (b), toluene (c) and 1-BuOH (d). (The peak at $2900 \mathrm{~cm}^{-1}$ is offset).

summarized here. As has been pointed out, ${ }^{6,25}$ cellulose including crystalline, amorphous parts and their interfaces is retained by planar-orientation or planarorientation-like structures (that is hydrophobic and hydrophilic planes). The hydrophobic parts are accumulated by intermolecular hydrogen bonds and the hydrophilic parts gather by van der Waals force, and both parts are interacted with each other. Such characteristic feature is intrinsically applicable to both celluloses having crystalline forms of cellulose I and cellulose II. Therefore, the ball-milling effect under the presence of solvents might work along the abovementioned planar orientation structure.

Now, for water, it has been known to be a good swelling agent to cellulose and decreased the hydrogen bonds between them. ${ }^{12-14}$ Water must be absorbed and localized in hydrophilic parts (or between hydrophilic planes) in the cellulose. The molecular mobility of cellulose in the parts containing water molecules is enhanced by the plasticizing effect of water, as has been pointed out on its $\tan \delta$-temperature analyses. ${ }^{26}$ Therefore, when the cellulose absorbing water received the external force, it is thought that the molecular chain slippage was caused at the interfaces between the hydrophilic parts. In this sense, the cellulose molecular aggregate parts containing water molecules could escape from the destruction of morphology and crystalline to some extent, rather leading to the transformation to cellulose II. On the other hand, the hydrophobic part of the cellulose without the plasticizing effect of water would mainly be subjected to the external stress. This might be related to the generation of cross-sectional cracks on cellulose microfibrils upon ball-milling.

In contrast, the inter- and intra-molecular hydrogen bonds of the cellulose are retained under the ball-milling in the presence of toluene or 1-butanol from IR results. The thermally stimulated current (TSC) measurements on cellulose/solvent systems have also revealed that the relaxation temperature for molecular parts controlled by the intermolecular hydrogen bonds of cellulose shifts to higher temperature under the existence of toluene. ${ }^{16,17}$ In this experiment, it can be said that the hydrophilic parts of the cellulose become stiff under the existence of toluene. Therefore, in contrast to the effect of water, the hydrophilic parts might be subjected to the impact and frictional stress by ballmilling. Cellulose molecules are piled up to microfibrils by inter-molecular hydrogen bonds and the microfibrils also interact with each other by inter-molecular hydrogen bonds. The peeling off phenomenon leading to the generation of the plate-like particles could be understood if it occurs due to the breakdown of the above-mentioned strong hydrogen bonds.

\section{The Adsorbing of a Dye Stuff on the Cellulose Parti-} cles.

The surface properties of the obtained particle were examined by using dye stuff with respect to the interaction of the solvent and cellulose. The cellulose particles prepared by the ball-milling with hydrophilic or hydrophobic solvent were mixed with a yellow dye stuff (4-aminoazobenzene, AAB) by ball-milling for an examination of anticipating the dye-accommodated 


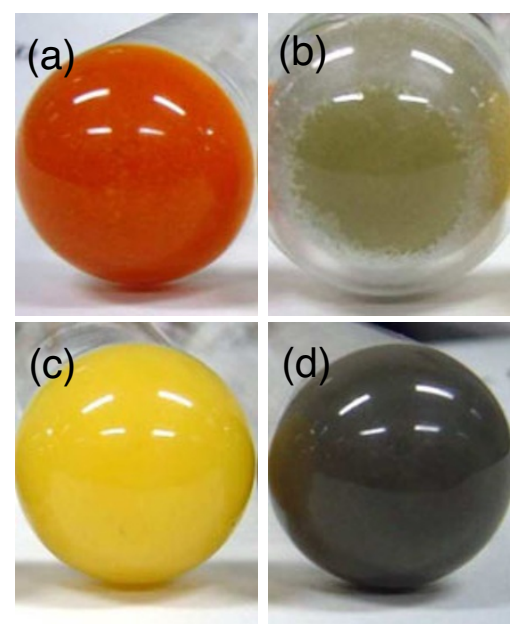

Figure 7. Optical photographs of the mixtures of the cellulose and $\mathrm{AAB}$ prepared by ball-milling, without solvent (a) and in presence of water (b), toluene (c) and 1-BuOH (d).

parts. The color development of the cellulose particles probably carries over the difference in interactions between the cellulose and each solvent. Since AAB is soluble in all kinds of solvents used here, the dye stuff in its cellulose mixtures would be adsorbed on the surface of the cellulose where each solvent infiltrated under the ball-milling. Here, AAB colored orange (or cinnabar) under acidic condition or yellow under neutral condition. ${ }^{24}$ The characteristic color development in the cellulose particle/dye stuff mixture was observed depending on the type of solvent used, a cinnabar; without solvent, a yellow; toluene and yellowish-green; water or 1-butanol (Figure 7). Note that $\mathrm{AAB}$ in each solvent gives almost yellow color and the milled $\mathrm{AAB}$ with solvent alone develops orange (or cinnabar) to slightly deep orange color, showing that the developed colors for the samples are derived mainly by the interaction with the cellulose. In addition, the UV-vis. spectrum for the solution of $\mathrm{AAB} /$ toluene was affirmed to be identical to those for the $\mathrm{AAB} /$ toluene solutions obtained after washing dye from the cellulose particle/dye samples, suggesting no chemical change of the dye stuff during the ballmilling.

Since the original cellulose contains some carboxyl group, if this portion of carboxyl group determines the color development of the cellulose/AAB mixtures, all the mixture should give orange color. However, the results were different as mentioned above. The cinnabar color developed by the ball-milling without solvent was due to the increase carboxyl group or free hydroxyl group. Although the water milling also leads to the slight increase of carboxyl group, the particle was not colored to cinnabar. Thus, the increased carboxyl group does not preferentially affect the color development of the cellulose/AAB mixture. The re- sults rather suggest that the color development is mainly determined by the parts in the cellulose where $\mathrm{AAB}$ is accommodated. The yellow color of the platelike particles by the toluene would indicate that $\mathrm{AAB}$ was adsorbed in a neutral or aprotic region, for instance in hydrophobic parts.

The disordered morphology of the particles with water and 1-BuOH millings was complicated to identify the region where $\mathrm{AAB}$ is absorbed, giving some strange colors. The color development in these two samples is difficult to explain logically, however, the possible explanation is made as follows. Supermolecular structures, such as crystallinity, molecular orientation, amorphous state and void fraction, have a strong influence on the sorption properties and the color development. Water and 1-butanol penetrated to inter-crystalline region (hydrogen bonding phase) and were absorbed there, cleaving some parts of hydrogen bonds (in the case of water processing) of the cellulose as was evidenced by the results of XRD and FT-IR. Therefore, the color development of these samples (b) and (d) in Figure 7 would be attributed to the heterogeneous structure mentioned above.

\section{CONCLUSIONS}

The solvents used here, i.e., water, toluene and 1-butanol affected the cellulose destruction under the ball-milling processing, giving the particular morphologies, polymorphs and crystallinity of the obtained cellulose particles. These differences in morphology and crystalline structure seemed to be attributed to the change of hydrogen bonds in the cellulose particles depending on the effect of the solvent. An experiment of the adsorbing of a dye stuff on the cellulose particles was carried out in order to seek for one of the surface on which a dye stuff is defused. The color development was discussed qualitatively in view of the surface property of the particle induced by the type of solvent used, under the ball-milling.

\section{REFERENCES}

1. For example, C. Yamane and K. Okajima, Sen-i Gakkaishi, 53, 321 (1997).

2. M. Ago, T. Endo, and T. Hirotsu, Cellulose, 11, 163 (2004).

3. H. Ono, M. Inamoto, and K. Okajima, Cellulose, 4, 57 (1997).

4. G. N. Marchenko, V. N. Marsheva, V. I. Kovalenko, Ye. M. Belova, G. M. Khrapovskii, N. G. Gustova, and V. F. Sopin, Polym. Sci. USSR, 25, 1647 (1983).

5. K. Sato, H. Mochizuki, K. Okajima, and C. Yamane, Polym. $J ., 36,478$ (2004).

6. C. Yamane, T. Aoyagi, M. Ago, K. Sato, K. Okajima, and T. Takahashi, Polym. J., 38, 819 (2006).

7. T. Hori and H. Zollinger, Text. Chem. Color., 18, 19 (1986). 
8. S. Woodcock, B. Henrissat, and J. Sugiyama, Biopolymers, 36, 201 (1995).

9. M. K. Inglesby, Ph.D.Dissertation, University of California, Davis, 2000.

10. A. Isogai and M. Usada, Sen-i Gakkaishi, 46, 324 (1990).

11. A. Isogai, M. Usada, T. Kato, T. Uryu, and R. H. Atalla, Macromolecules, 22, 3168 (1989).

12. A. J. Stamm, "Wood and Cellulose Science," Ronald Press, New York, 1964, Chapter 7 and 8.

13. J. Nakano, T. Higuchi, M. Sumimoto, and A. Ishidu, "Mokuzai-Kagaku," Uni Publishers, Tokyo, 1985, Chapter 2.

14. S. P. Rowland, ACS Symposium Series No. 49, 1977, Chapter 2.

15. Jpn.-open patent 2004-230719.

16. M. Sasahira, C. Yamane, and K. Okajima, Cell. Commun., 10, 2 (2003).

17. C. Yamane, M. Mori, M. Saito, and K. Okajima, Polym. J.,
28, 1039 (1996).

18. "Cellulose and Cellulose Derivatives," E. Otto Ed., Interscience Publishers, Inc, 1954, Vol. IV chapter XIII.

19. C. Y. Liang and R. H. Marchessult, J. Polym. Sci., 37, 385 (1959).

20. N. V. Ivanova, E. A. Korolenko, E. V. Korolik, and R. G. Abankov, Zh. Prikl. Spektrosk., 51, 301 (1989).

21. D. Fengel, Holzforschung, 47, 103 (1993).

22. Y. Maréchal and H. Chanzy, J. Mol. Struct., 523, 183 (2000).

23. Y. Maréchal, J. Chem. Phys., 87, 6344 (1987).

24. K. Bredereck, W. A. Schick, and E. Bader, Makromol. Chem., 186, 1643 (1985).

25. M. Ago, K. Sato, and K. Okajima, Cellulose Commun., 12, 58 (2005).

26. S. Manabe, M. Iwata, and K. Kamide, Polym. J., 18, 1 (1986). 